\title{
Avaliação da dor e morbidade local da retirada do enxerto ósseo da crista ilíaca para artrodese cervical anterior
}

Evaluation of the pain and local morbidity of the insertion taken out from the iliac crest to the anterior cervical arthrodesis

\author{
Evaluación del dolor y morbilidad local del injerto extraído de la \\ cresta ilíaca para artrodesis cervical anterior
}

\author{
Rafael Barreto Silva' \\ Paulo Tadeu Maia Cavali² \\ Ivan Guidolin Veiga ${ }^{3}$ \\ Marcelo Italo Risso-Neto ${ }^{3}$ \\ Wagner Pasqualini ${ }^{3}$ \\ Marcus Alexandre Mello Santos ${ }^{4}$ \\ Alexander Junqueira Rossato ${ }^{4}$ \\ Maurício Antonelli Lehoczki ${ }^{4}$ \\ Elcio Landim 5
}

\section{RESUMO}

Objetivo: avaliar as possíveis complicações associadas à retirada de enxerto da crista ilíaca anterior em cirurgia para artrodese cervical anterior, em especial a dor residual. Métodos: foi realizado estudo retrospectivo com análise de prontuários e aplicação de questionário via telefone com 20 pacientes no período compreendido entre Agosto de 2008 e Novembro de 2009. Todos os pacientes foram submetidos à mesma técnica cirúrgica para extração do enxerto, sendo operados pela mesma equipe no Hospital de Clinicas da Unicamp (HC Unicamp). As variantes analisadas foram dor

\section{ABSTRACT}

Objective: to evaluate the possible complications associated to the removal of the insertion of the anterior iliac crest in surgery to the anterior cervical arthrodesis, especially to the residual pain. Methods: the retrospective study with the medical records analysis and the application of a form by telephone with 20 patients in a period from August 2008 to November 2009 were performed. All patients were submitted to the same surgical technique for the crest extraction, and were operated by the same team at Hospital das Clinidas da Unicamp (HC Unicamp). The

\section{RESUMEN}

Objetivo: evaluar las posibles compilaciones asociadas a la retirada del injerto de la cresta ilíaca anterior en cirugía para artrodesis cervical anterior, principalmente los dolores residuales. Métodos: fue realizado un estudio retrospectivo con análisis de prontuarios y con aplicación de cuestionario por teléfono para 20 pacientes entre agosto de 2008 $y$ noviembre de 2009. Todos los pacientes fueron sometidos al mismo procedimiento cirúrgico para la retirada del injerto, siendo que fueron operados por el mismo equipo en el Hospital de Clínicas de la Unicamp (HC Unicamp). Las variantes anali-

\footnotetext{
Trabalho realizado no Grupo de Cirurgia de Coluna Vertebral da Universidade Estadual de Campinas - UNICAM) - Campinas (SP), Brasil.

'Estagiário da Disciplina de Cirurgia de Coluna do Departamento de Ortopedia e Traumatologia da Universidade Estadual de Campinas - UNICAMP - Campinas (SP), Brasil; e do Grupo de Escoliose da Associação de Assistência à Criança Deficiente - AACD - São Paulo (SP), Brasil.

2 Médico Assistente da Disciplina de Cirurgia de Coluna do Departamento de Ortopedia e Traumatologia da Universidade Estadual de Campinas - UNICAMP Campinas (SP), Brasil; e do Grupo de Escoliose da Associação de Assistência à Criança Deficiente - AACD - São Paulo (SP), Brasil.

${ }_{3}^{3}$ Médico Assistente da Disciplina de Cirurgia de Coluna do Departamento de Ortopedia e Traumatologia da Universidade Estadual de Campinas - UNICAMP Campinas (SP), Brasil.

${ }^{4}$ Médico Assistente do Grupo de Escoliose da Associação de Assistência à Criança Deficiente - AACD - São Paulo (SP), Brasil.

${ }_{5}^{5}$ Professor da Disciplina de Cirurgia de Coluna do Departamento de Ortopedia e Traumatologia da Faculdade de Ciências Médicas da Universidade Estadual de Campinas - UNICAMP - Campinas (SP), Brasil; e Chefe do Grupo de Escoliose da Associação de Assistência à Criança Deficiente - AACD - São Paulo (SP), Brasil.
} 
residual, taxa de infecção, lesão neurológica ou vascular e ocorrência de fratura da asa do ilíaco. Os dados foram colocados em uma tabela e as médias e porcentagens foram calculadas. Resultados: dos 20 pacientes, 12 homens e 8 mulheres, com média de idade de 51,75 anos (29-74) e follow-up médio de 11,83 meses (2-29), não houve nenhuma lesão grave, como fratura, lesão arterial ou neurológica. Houve um caso de infecção superficial $(5 \%)$ e $25 \%$ dos pacientes queixaram-se de desconforto leve e dificuldade para deambular não incapacitante. Conclusão: a retirada de enxerto da crista ilíaca anterior está associada a muitas complicações, sendo importante o conhecimento de outras opções de enxerto e exposição ao paciente das possíveis complicações. Por meio deste levantamento, não verificamos nenhuma complicação grave, e o percentual de pacientes com dor residual acompanha os achados na literatura, podendo ser diminuído com uma dissecção cuidadosa da crista ilíaca.

DESCRITORES: Artrodese; Enxerto ósseo; Comorbidades; Dissecção

variants analyzed were: residual, pain infection rate, neurologic or vascular lesion and occurrence of the fracture of the iliac wing. The data was placed in a table and the average and percentage were calculated. Results: of the 20 patients, 12 men and 8 women, with average age of 51.75 years (2974) and mean follow-up of 11,83 months (2-29), we did not find any serious lesion like fracture, arterial or neurologic lesion. There was a case of superficial infection (5\%), and $25 \%$ of the patients complained about light discomfort and not incapacitating difficulty to prowl. Conclusion: the removal of the anterior iliac crest is associated to many complications. It is important to know of other insertion options and make the patient aware of the possible complications. Through this research, we did not find any serious complications, and the patients' percentage with residual pain are the same as in the literature, and can be diminished through a careful dissection of the iliac crest.

KEYWORDS: Arthrodesis; Bone insertion; Comorbidity; Dissection zadas fueron: dolor residual, tasa de infección, lesión neurológica o vascular y aparición de fractura en el ala ilíaca. Los datos fueron colocados en una tabla, y las mediciones y porcentajes fueron calculados. Resultados: de los 20 pacientes, 12 hombres y 8 mujeres, con edad media de 51,75 años (29-74), acompañamiento medio de 11,83 meses (2-29). No tuvo ninguna lesión grave como fractura, lesión arterial o neurológica. Hubo un caso de infección superficial (5\%), y $25 \%$ de los pacientes se quejaron de malestar leve y dificultades para andar no incapacidad. Conclusión: la retirada del injerto de la cresta ilíaca anterior esta relacionada a muchas complicaciones, siendo importante el conocimiento de otras opciones de injerto y exposición al paciente de las posibles complicaciones. A través del levantamiento, no se verificó ninguna complicación grave y el porcentual de pacientes con dolor residual pude se comparar con los encontrados en literatura, pudiendo ser reducidos a través de una disección cuidadosa de la cresta ilíaca.

DESCRIPTORES: Artrodesis; Injerto Óseo; Co-Morbilidad; Disección

\section{INTRODUÇÃO}

O enxerto autólogo da crista ilíaca é utilizado em cirurgias ortopédicas e nas artrodeses espinhais com o objetivo de reconstruir a coluna anterior, restaurar a lordose e a capacidade de sustentação de cargas e promover a fusão óssea ${ }^{1}$. As vantagens do enxerto autógeno em relação ao aloenxerto ou enxertos sintéticos incluem propriedades osteoindutoras e osteocondutoras superiores, além de existir um risco menor de transmissão de doenças, bem como de rejeição do mesmo devido à menor imunogenicidade em relação ao enxerto alógeno ${ }^{1}$. Os relatos de comorbidades decorrentes da retirada de enxerto ósseo da crista ilíaca em cirurgias espinhais são inúmeros na literatura, tanto no acesso anterior como no posterior à $\operatorname{crista}^{1-10}$. As principais queixas são: dor crônica, lesão neurológica do nervo cutâneo lateral femoral no acesso anterior à crista e do nervo ciático e/ ou clúneos no acesso posterior, lesão vascular, fratura e infecção. Na literatura, os relatos de complicações variam de 3 a $61 \%{ }^{5}$. Devido a essas comorbidades, muitos autores descrevem novas técnicas de retirada de enxerto para minimizar a agressão à crista ilíaca e aos tecidos adjacentes ${ }^{10}$. Foram criados miniacessos com dissecção cuidadosa dos tecidos ${ }^{10}$, técnicas de reconstrução da crista após obtenção do enxerto ${ }^{2,11} \mathrm{e}$, atualmente, a utilização de substitutos ao enxerto de ilíaco, que ainda são considerados gold standard, com taxas de fusão superiores a $90 \%$ na maioria dos estudos ${ }^{1}$.

O objetivo do trabalho foi realizar um levantamento retrospectivo no HC da Unicamp para comparar nossos resultados com a literatura.

\section{MÉTODOS}

O estudo compreendeu um levantamento de todos os pacientes operados no período de Agosto de 2008 a Novembro de 2009 no Serviço de Cirurgia da Coluna do Hospital de Clínicas da Universidade Estadual de Campinas - Unicamp. Foram selecionados aqueles submetidos à artrodese cervical via anterior, onde foi realizada a retirada de enxerto da crista ilíaca. Nessa seleção foram 
encontrados 20 pacientes, sendo então realizada uma revisão de todos os prontuários e entrevista via telefone com 20 pacientes para completar o questionário. Variantes como dor, infecção, lesão neurológica ou vascular e fratura da crista ilíaca foram questionadas e classificadas como agudas quando ocorreram em menos de três meses, ou crônicas com mais de três meses. $\mathrm{O}$ follow-up médio foi de 11,83 meses (2 a 29), com 12 homens e 8 mulheres, e média de idade de 51,75 (29 a 74).

Pacientes com dor persistente foram questionados sobre a intensidade da dor. A dor foi quantificada como leve, moderada ou grave, conforme a necessidade de uso de analgésicos e limitações na vida diária. Os dados dos pacientes que responderam a todo o questionário foram colocados em uma tabela, e as médias e porcentagens foram calculadas.

Na técnica cirúrgica de retirada de enxerto da crista ilíaca anterior, realizamos a palpação e marcação da espinha ilíaca ântero-superior (EIAS), medimos $3 \mathrm{~cm}$ a partir desse ponto, diminuindo, assim, o risco de lesão neurológica e de fratura da crista ilíaca. Delimitamos a espessura da mesma e desenhamos a incisão na pele, a qual varia conforme os níveis de artrodese envolvidos (Figura 1).

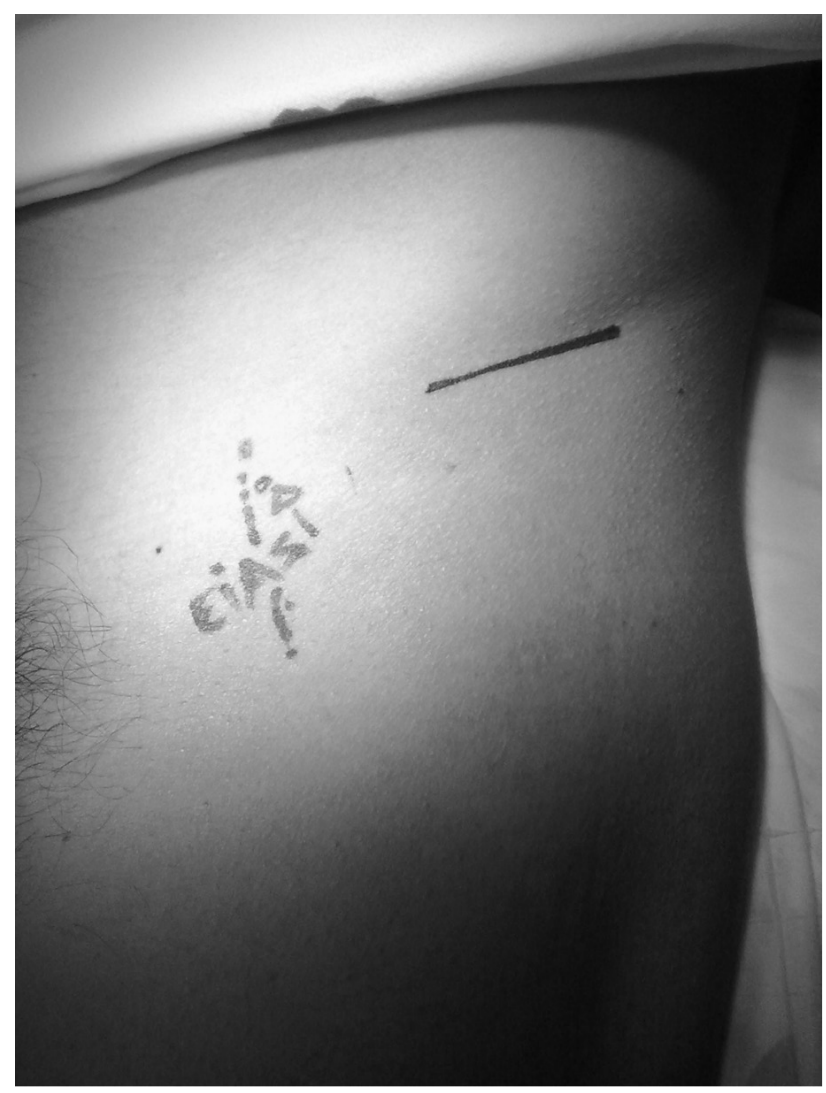

Figura 1

Marcação na pela da incisão $3 \mathrm{~cm}$ posteriores a EIAS.

Antes da realização da incisão, infiltramos a pele com anestésico local (Figura 2), bupivacaína 7,5\%.

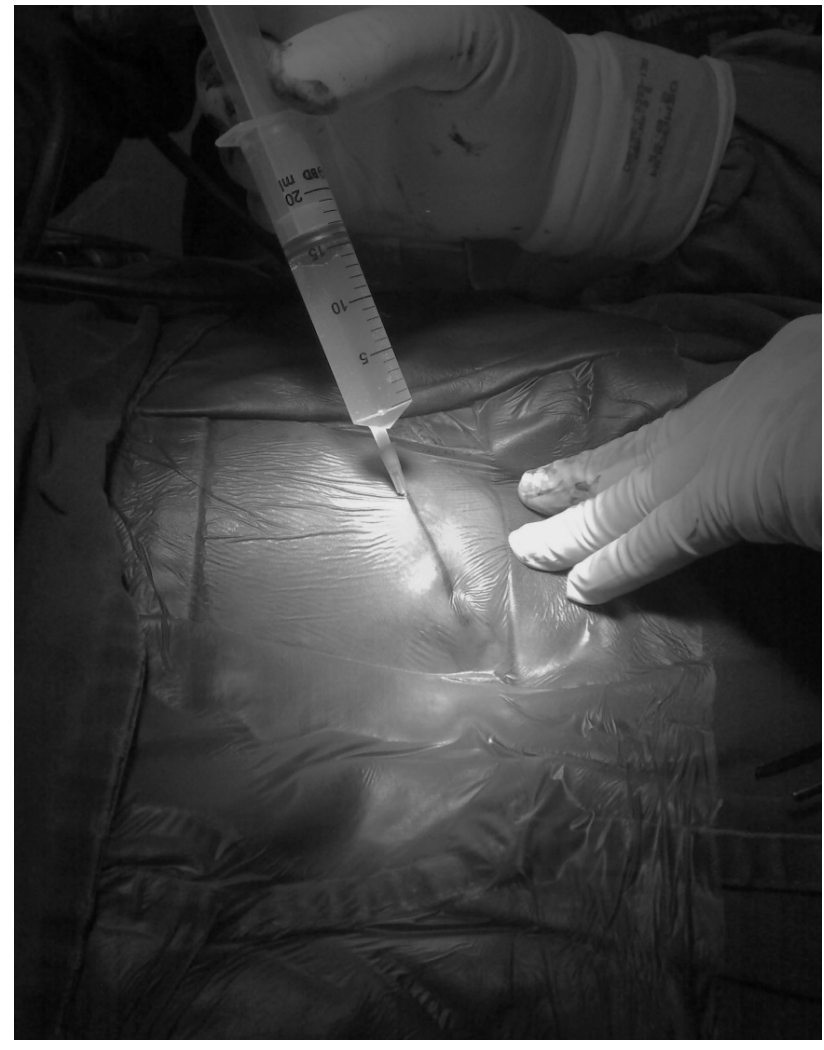

Figura 2

Infiltração da pele com bupivacaína 7,5\%.

É realizada a incisão e uma discecção cuidadosa dos tecidos com exposição subperiostal da crista (Figura 3). A retirada do enxerto é feita com um formão faca $(2 \mathrm{~cm})$, que faz o primeiro corte na crista, deixado no local para manter a tensão do osso e evitar fraturas na retirada do fragmento trapezoidal tricortical, que é feita com um formão $1 / 4$ de polegada (Figura 4 A e B).

Uma hemostasia cuidadosa seguida de lavagem da ferida operatória foi feita, e é feita a colocação de cera para osso como complementação da hemostasia. É colocado dreno de Portovac $\AA$ de $3,2 \mathrm{~mm}$, realizado o fechamento por planos dos tecidos e um curativo provisório compressivo (Figura 5).

\section{RESULTADOS}

No período analisado, foram realizadas 20 cirurgias com discectomia cervical anterior e fusão com retirada de enxerto da crista ilíaca anterior. Doze pacientes eram do sexo masculino (60\%) e oito do sexo feminino (40\%). A média de idade foi de 51,75 anos,variando de 29 a 74 anos, e o follow-up médio foi de 11,83 meses, variando de 2 a 29 meses. Não tivemos nenhuma complicação grave, como fratura da crista ilíaca, lesão arterial ou neurológica. Um paciente $(5 \%)$ apresentou infecção superficial da ferida operatória necessitando de curativos diários e uso prolongado de cefalexina por 10 dias. Em relação à dor residual, cinco pacientes (25\%) referiram ter dor leve no sítio doador com desconforto e quatro $(20 \%)$ referiram dor ao deambular, porém, sem limitar suas atividades diárias. 


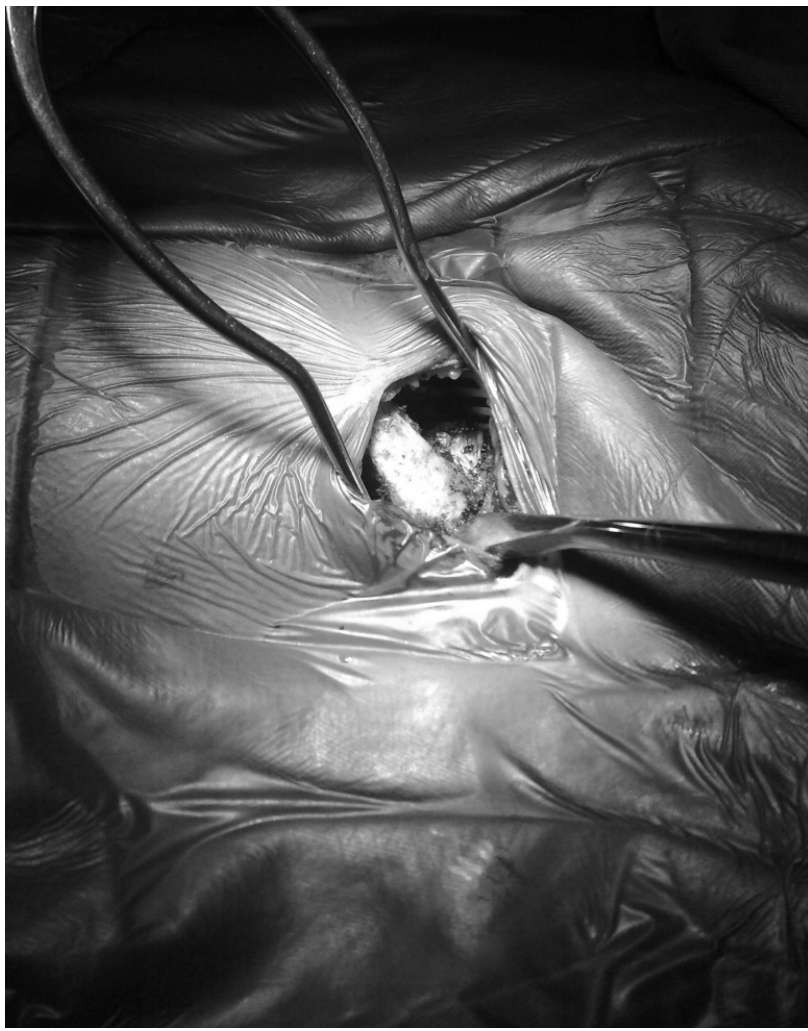

Figura 3

Exposição subperiosteal da crista ilíaca.

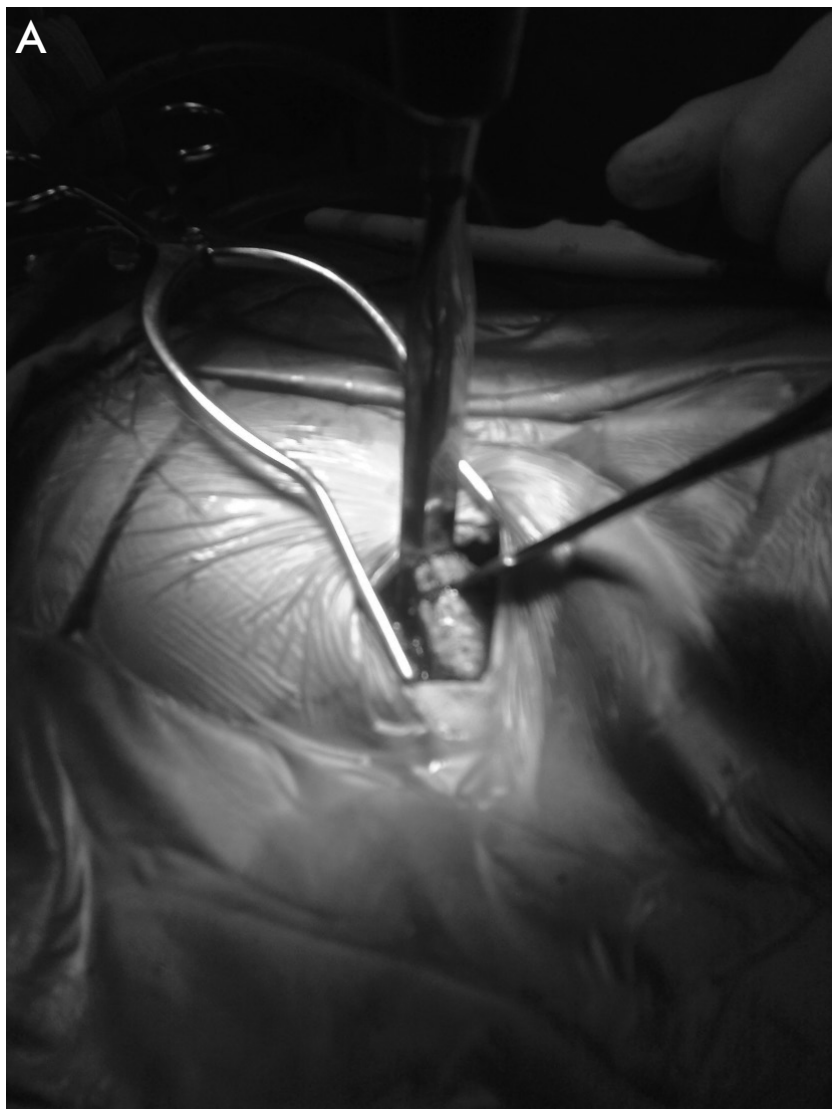

Figura 4 A e B

Retirada do enxerto com formão.

\section{DISCUSSÃO}

O princípio básico da maioria das cirurgias espinhais inclui uma descompressão adequada e utilização de enxerto ósseo para promover a restauração da integridade estrutural e alcançar uma sólida fusão óssea ${ }^{1}$. As vantagens teóricas do enxerto autólogo incluem: altas taxas de fusão, diminuição das taxas de colapso e evita os riscos de transmissão de doenças ${ }^{1}$. Quando o enxerto da crista ilíaca é usado, possíveis morbidades no sítio doador podem ocorrer $^{1-10}$. O presente estudo demonstrou que uma dissecção adequada e cuidadosa da crista ilíaca anterior, respeitando os parâmetros anatômicos, diminui a chance de complicações no sítio doador. Nossos achados estão de acordo com os resultados de outros trabalhos da literatura e evidenciaram $25 \%$ de dor residual local de leve intensidade sem incapacitação para deambular, um caso de infecção superficial que necessitou uso prolongado de antibiótico e nenhuma complicação maior, como fratura da crista, lesão arterial ou neurológica. Esses achados foram comparados com outros estudos reportados na literatura.

Em um estudo anatômico de 50 cristas ilíacas bilaterais, Smucker et al. ${ }^{12}$ fizeram uma análise morfométrica do ilíaco humano avaliando a distância da incisura ciática (PN), distância da articulação sacroilíaca (PS), medida ântero-posterior da incisura ciática e máxima distância da articulação sacroilíaca. Eles evidenciaram que as maiores

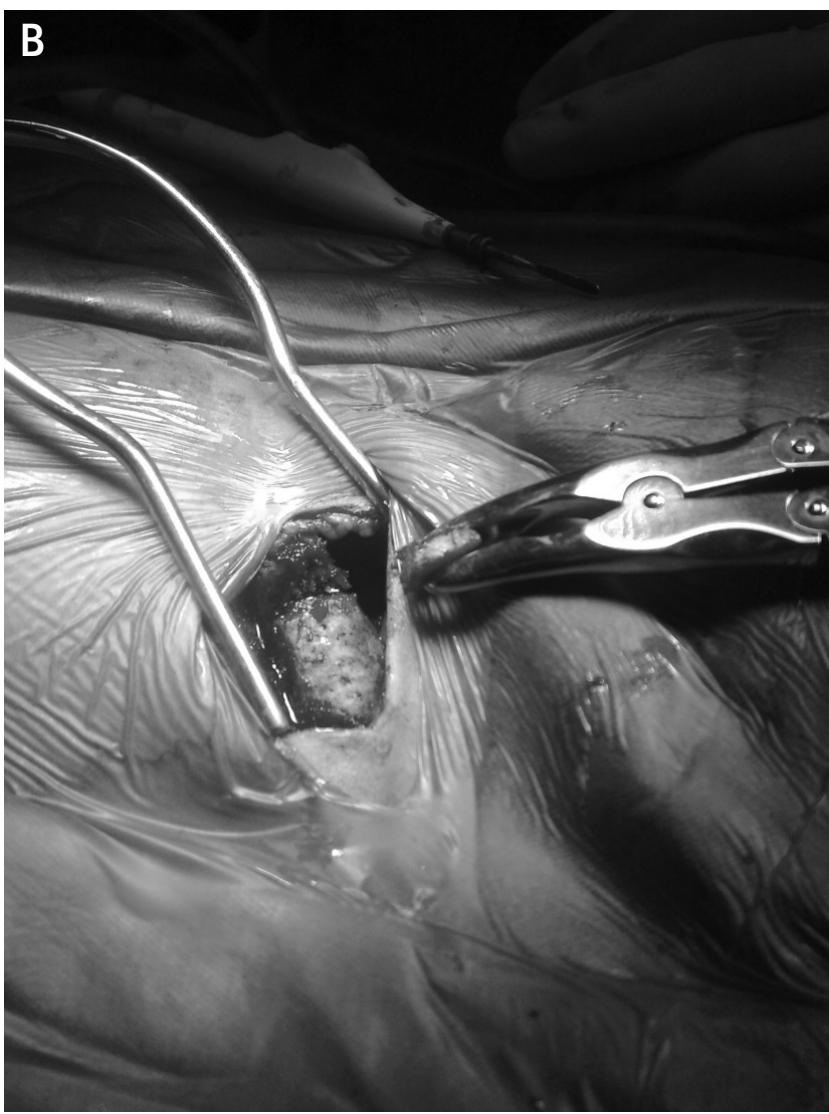




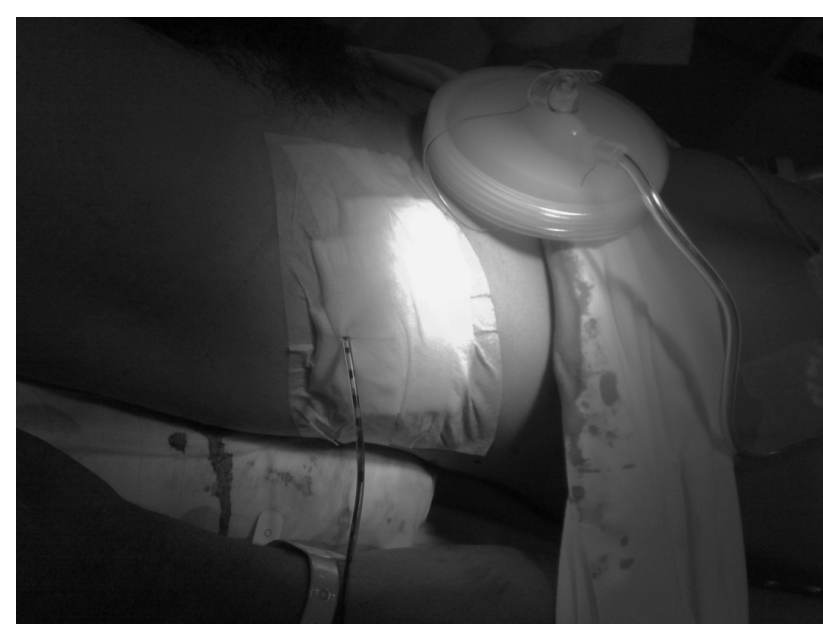

Figura 5

Curativo com dreno de Portovac ${ }^{\circledR} 3,2$.

distâncias são encontradas nos homens: PN homens de $43,7 \mathrm{~mm}( \pm 4,6 \mathrm{~mm})$, PN mulheres de $39,7 \mathrm{~mm}( \pm 5,8 \mathrm{~mm})$ e PS homens de $21,5 \mathrm{~mm}$ versus $16,9 \mathrm{~mm}$ nas mulheres. Em outro estudo anatômico, Mischkowski et al. ${ }^{13}$ descreveram o trajeto do nervo cutâneo femoral lateral e verificaram que, em 34 cadáveres, o nervo passava acima da EIAS em $1 \%$ dos casos a uma distância menor de $5 \mathrm{~mm}$ lateral. Relataram 298 pacientes que sofreram retirada de enxerto da crista ilíaca anterior, com quatro $(1,3 \mathrm{a} \%)$ apresentando alterações sensitivas, e reforçaram a necessidade de dissecção cuidadosa da crista. Ebraheim et al. ${ }^{14}$ verificaram que a melhor região para retirada de enxerto fica localizada a $3 \mathrm{~cm}$ posteriores à EIAS, sendo essa região um sítio doador tanto para enxertos bicorticais como para tricorticais.

Nos trabalhos de Spallone et al. ${ }^{15}$ e David et al. ${ }^{7}$, a técnica cirúrgica influencia os resultados pós-operaatórios. Spallone defende uma dissecção cuidadosa com exposição subperiostal do ilíaco, evitando o descolamento da musculatura anterior e levando à melhores resultados.

Em um estudo prospectivo, Acharya et al. ${ }^{2}$ tentam validar a hipótese de que a reconstrução da crista diminui a dor no sítio doador. O estudo foi feito em 26 pacientes dos quais foi retirado enxerto tricortical com defeito, e reconstruído com o substituto Chifra $\mathrm{HABG}^{\circledR}$, com seguimento de um ano e avaliação clínica e por meio de imagem radiográfica. $\mathrm{O}$ estudo concluiu que a reconstrução do sítio doador diminui a dor local e que o Chifra $\mathrm{HABG}^{\circledR}$ é uma boa opção para reconstrução.

Defino et al. ${ }^{11}$ relatam uma técnica de reconstrução da crista com costela retirada do acesso anterior à coluna vertebral de 15 pacientes, com bom resultado clínico radiográfico e estético.

Sasso et al. ${ }^{3}$, em um estudo multicêntrico controlado prospectivo, avaliaram 202 pacientes e verificaram que dois (1\%) não tiveram dor, e que 33\%, após um ano, ainda apresentavam dor no sítio doador. Esse trabalho confirma que o enxerto de ilíaco é continua sendo o Gold Standard, mas está frequentemente associado à dor e complicações no pós-operatório (2,8 a 39\%).
Em um estudo retrospectivo para avaliar complicações em crianças, Skaggs et al. ${ }^{4}$ aplicaram um questionário com 87 pacientes de um total de 214 , e evidenciaram uma lesão arterial $(0,5 \%)$, duas infecções ( $1 \%), 24 \%$ com dor e $14 \%$ com problemas para realizar atividades diárias.

Banwart et al. ${ }^{5}$ avaliaram causas, taxas e fatores de risco para morbidade em 261 pacientes, e classificaram as complicações em menores, maiores, agudas ou crônicas. Nesse estudo, encontraram 10\% dos casos com dor e 39\% de menores complicações, que podem ser evitadas com a dissecção cuidadosa dos tecidos.

Complicações relativas à retirada de enxerto do ilíaco posterior também são bem documentadas na literatura. Delawi et al. ${ }^{6}$ em um estudo de coorte retrospectivo em pacientes que sofreram fraturas traumáticas na coluna e que foram submetidos à artrodese e fusão com osso da crista ilíaca evidenciaram que $14,3 \%$ dos que foram submetidos à artrodeses altas (T12 a L2) reportaram dor no sítio doador, comparados com os pacientes submetidos a intervenções baixas, até L3 ou mais caudalmente, (40,9\%), demonstrando como conclusão a incapacidade dos pacientes de diferenciar o local doloroso e a provável superestimação da dor na área doadora do ilíaco posterior.

David $^{7}$ relata que a dor, a sensibilidade e as alterações sensoriais são muito frequentes, e compara a retirada de enxerto posterior através da mesma incisão mediana versus uma segunda incisão (56 pacientes no grupo mesma incisão e 51 no grupo outra incisão) e constata que no grupo mesma incisão ocorreu menos dor, menor sensibilidade e menor risco de alterações sensoriais.

Robertson et al. ${ }^{8}$, fizeram um estudo prospectivo com 106 pacientes submetidos à fusão espinhal posterior para avaliar a morbidade no sítio doador. Os pacientes foram avaliados 3,6 e 12 meses após a cirurgia, e os resultados mostraram significativas diferenças na morbidade nos diferentes níveis operados, sendo que os resultados na coluna lombossacra são piores que na coluna cervical e toracolombar.

Kim et al. ${ }^{9}$ avaliaram prospectivamente 110 pacientes submetidos à fusão lombar eletiva com avaliação em 6 semanas, 10 meses e 12 meses, concluindo que a dor crônica é a complicação mais importante. Em um ano, 15,1\% apresentavam dor, $15,1 \%$ dificuldade para deambular, $5,2 \%$ apresentavam limitação no trabalho, $12,9 \%$ para recreação, $14,1 \%$ para tarefas diárias, $7,6 \%$ com problemas na vida sexual e $5,9 \%$ com irritação pelas roupas.

Em um trabalho retrospectivo, Silber et al. ${ }^{1}$ avaliaram por meio de questionário 187 pacientes submetidos à artrodese lombar de um nível e evidenciaram que 50\% apresentavam dificuldade para deambular, 26,1\% tinham dor no sítio doador (EVA médio 3,8), 11,2 tinham necessidade de uso crônico de analgésicos, além de outras complicações pós-operatórias precoces.

\section{CONCLUSÃO}

O enxerto autólogo continua sendo o gold standard, promovendo uma ideal combinação entre alta osteoindução e 
osteocondução, bom suporte estrutural e baixa imunogenicidade. Sua retirada está associada a inúmeras complicaçõe,s que devem ser discutidas com os pacientes, e as outras opções de enxertos devem ser lembradas. Com esse levantamento, não verificamos nenhuma complicação grave, e o percentual de pacientes com dor residual acompanha os achados na literatura, que pode ser diminuído através de uma dissecção cuidadosa da crista ilíaca.

\section{REFERÊNCIAS}

1. Silber JS, Anderson DG, Daffner SD, Brislin BT, Leland JM, Hilibrand AS, et al. Donor site morbidity after anterior iliac crest bone harvest for single-level anterior cervical discectomy and fusion. Spine (Phila Pa 1976). 2003;28(2):134-9.

2. Acharya NK, Mahajan CV, Kumar RJ, Varma HK, Menon VK. Can iliac crest reconstruction reduce donor site morbidity?: a study using degradable hydroxyapatite-bioactive glass ceramic composite. J Spinal Disord Tech. 2010;23(4):266-71.

3. Sasso RC, LeHuec JC, Shaffrey C; Spine Interbody Research Group. Iliac crest bone graft donor site pain after anterior lumbar interbody fusion: a prospective patient satisfaction outcome assessment. J Spinal Disord Tech. 2005;18 Suppl:S77-81.

4. Skaggs DL, Samuelson MA, Hale JM, Kay RM, Tolo VT. Complications of posterior iliac crest bone grafting in spine surgery in children. Spine (Phila Pa 1976). 2000;25(18):2400-2.

5. Banwart JC, Asher MA, Hassanein RS. Iliac crest bone graft harvest donor site morbidity. A statistical evaluation. Spine (Phila Pa 1976). 1995;20(9):1055-60.
6. Delawi D, Dhert WJ, Castelein RM, Verbout AJ, Oner FC. The incidence of donor site pain after bone graft harvesting from the posterior iliac crest may be overestimated: a study on spine fracture patients. Spine (Phila Pa 1976). 2007;32(17):1865-8

7. David R, Folman Y, Pikarsky I, Leitner Y, Catz A, Gepstein R. Harvesting bone graft from the posterior iliac crest by less traumatic, midline approach. J Spinal Disord Tech. 2003;16(1):27-30.

8. Robertson PA, Wray AC. Natural history of posterior iliac crest bone graft donation for spinal surgery: a prospective analysis of morbidity. Spine (Phila Pa 1976). 2001;26(13):1473-6.

9. Kim DH, Rhim R, Li L, Martha J, Swaim BH, Banco RJ, et al. Prospective study of iliac crest bone graft harvest site pain and morbidity. Spine J. 2009;9(11):886-92.

10.Pollock R, Alcelik I, Bhatia C, Chuter G, Lingutla K, Budithi C, et al. Donor site morbidity following iliac crest bone harvesting for cervical fusion: a comparison between minimally invasive and open techniques. Eur Spine J. 2008;17(6):845-52.

11.Defino HL, Rodriguez-Fuentes AE. Reconstruction of anterior iliac crest bone graft donor sites: presentation of a surgical technique. Eur Spine J. 1999;8(6):491-4.
12.Smucker JD, Akhavan S, Furey C. Understanding bony safety zones in the posterior iliac crest: an anatomic study from the Hamann-Todd collection. Spine (Phila Pa 1976). 2010;35(7):725-9.

13.Mischkowski RA, Selbach I, Neugebauer J, Koebke J, Zöller JE. Lateral femoral cutaneous nerve and iliac crest bone grafts--anatomical and clinical considerations. Int J Oral Maxillofac Surg. 2006;35(4):366-72.

14.Ebraheim NA, Yang H, Lu J, Biyani A, Yeasting RA. Anterior iliac crest bone graft. Anatomic considerations. Spine (Phila Pa 1976). 1997;22(8):847-9.

15.Spallone A. A less-invasive technique for harvesting autologous iliac crest grafts for cervical interbody fusion: technical note. Surg Neurol. 2007;67(2):160-2.

\section{Correspondência}

Rafael Barreto Silva

Departamento de Ortopedia e Traumatologia da Universidade Estadual de Campinas - UNICAMP - Campinas (SP), Brasil

Rua: Tessália Vieira de Camargo, 126

- Cidade Universitária Zeferino Vaz

CEP: 13083-887

E-mail: rafaelbarreto.coluna@gmail.com 